\title{
Foundations of Meissner Superconductor Magnet Mechanisms Engineering
}

\author{
Jose Luis Perez-Diaz and Efren Diez-Jimenez \\ Dpt. Ingeniería Mecánica - Universidad Carlos III de Madrid \\ Spain
}

\section{Introduction}

It has long been known that a repulsive force arises between a magnetic field (generated, for instance, by a permanent magnet - PM) and a superconductor -Sc (Arkadiev, 1947). This force is due to the repulsion of the magnetic field away from the superconductor the Meissner effect. Type I superconductors only can be in the Meissner state, which means that a magnetic field will be always expelled from the superconductor, independently of its poles orientation. Nevertheless, type II superconductors may be in two different states: first, provided the magnetic field is low enough, they are at a Meissner state similar to type I superconductors. In this Meissner state they absolutely expel the magnetic field and prevalent repulsive forces appear. Second, for magnetic fields larger than the so-called First Critical Field $\mathrm{H}_{\mathrm{C} 1}$, the magnetic flux penetrates the superconductor creating a magnetization which contributes to an attractive resulting force. This second state is known as mixed state.

In 1953 Simon first tried to make a superconducting bearing (Simon, 1953) using superconductors in the mixed state.The first engine using a superconducting bearing was made in 1958 (Buchhold, 1960). After the discovery of high critical temperature superconductors (Bednorz \& Müller, 1986), the Meissner repulsive force has become a popular way of demonstrating superconducting properties (Early et al., 1988).For calculating forces between a magnet and a superconductor it is necessary to have models that describe both the flux penetration state and the Meissner state repulsion. The first one can be solved by using conventional methods to compute forces between magnetic elements and magnetized volumes. However, for the Meissner state the question has remained open until these last years.

Several models using the method of images to calculate superconducting repulsion forces (Lin, 2006; Yang \& Zheng, 2007) have been proposed. However, this method of images is limited to a few geometrical configurations that can be solved exactly, and the physical interpretation of the method is under discussion (Giaro et al., 1990; Perez-Diaz \& GarciaPrada, 2007). Furthermore, some discrepancies within experiments still exist (Hull, 2000).

A general local model based on London's and Maxwell's equations has been developed to describe the mechanics of the superconductor-permanent magnet system (Perez-Diaz et al., 2008). Due to its differential form, this expression can be easily implemented in a finite elements analysis (FEA) and is consequently appliable to any shape of superconductor in pure Meissner state (Diez-Jimenez et al. 2010). 
In this chapter, we present the demonstration of the model, the implementation into a finite elements program, the experimental verification and its limit of application. To finish, we show an example of usage.

\section{Magneto-mechanics of a superconductor in Meissner state}

A superconductor is in a pure Meissner state when it is exposed to an externally applied magnetic field, $\vec{H}^{a p}$, lower than a certain value, $\mathrm{H}_{\mathrm{C} 1}$. $\mathrm{H}_{\mathrm{C} 1}$ is a characteristic of the material (Alario \& Vincent, 1991), which depends on temperature. In this case, it is assumed that both $\vec{H}$ and $\vec{B}$ are equal to zero inside the superconductor. When a magnetic field is then applied -for example by moving a permanent magnet close to the superconductor - a surface current density is generated on the outermost surfaces. According to the London equation, this current is confined only to a depth of $\lambda(\mathrm{T})$. Type II superconductors, such as the rare earth oxide high temperature superconductors, have the highest values for $\lambda$, reaching typical values of thousands of Amstrongs (Umezawa \& Crabtree, 1998). Therefore, as this paper deals with macroscopic elements, it can be approximated that current density has an infinitely localized surface current

$$
\vec{J}=\vec{j}_{s}(x, y) \delta(z)
$$

where $\vec{j}_{s}(x, y)$ is a surface current density tangent to the surface vector field and $\delta(z)$ is a Dirac delta function on $z$. This current density will make $\vec{H}$ discontinuous when passing from the air or vacuum $(z>0)$ into the superconductor $(z<0)$. The second Maxwell law (Jackson, 1975) relates the magnetic field and the current density in such a way that $\vec{j}_{s}(x, y)$ is determined by $\vec{H}^{a p}$.Using units from the MKSA system, this second Maxwell law can be written as

$$
\vec{\nabla} \times \vec{H}=\vec{j}+\frac{\partial \vec{D}}{\partial t}
$$

In the static limit it can be assumed that $\frac{\partial \vec{D}}{\partial t}=0$. Therefore it may simply be written as:

$$
\vec{\nabla} \times \vec{H}=\vec{J}
$$

$\vec{H}$ may be decomposed in that externally applied $\vec{H}^{a p}$ and that generated by the superconducting currents $\vec{H}^{s c}$. Furthermore, these three vector fields will be decomposed both in tangent and normal to the surface components:

$$
\vec{H}_{/ /}=\vec{H}_{/ /}^{a p}+\vec{H}_{/ /}^{s c}
$$

and

$$
\vec{H}_{\perp}=\vec{H}_{\perp}^{a p}+\vec{H}_{\perp}^{s c}
$$


Note that $\vec{H}^{a p}$ is continuous and fulfils: $\vec{\nabla} \times \vec{H}^{a p}=0$, provided the permanent magnet does not touch the superconductor surface. On the contrary, both $\vec{H}$ and $\vec{H}^{s c}$ are discontinuous at the superconducting surface. In particular, both $H_{/ /}$and $H_{/ /}^{s c}$ are discontinuous.

By using the divergence theorem (Jackson, 1975) on a small parallelepiped with volume $V$, a face just above the superconductor surface and another parallel face under it, it can be written that:

$$
\int_{V}(\vec{\nabla} \times \vec{H}) d^{3} x=\int_{S}\left(\vec{n}_{s} \times \vec{H}\right) d S
$$

where $S$ is the surface defining this parallelepiped and $n_{S}$ its normal vector. By using Maxwell law (3) it can be reduced to:

$$
\int_{V} \vec{J} d^{3} x=\int_{S}\left(\vec{n}_{s} \times \vec{H}\right) d S
$$

But, taking (1) into account, and considering $\vec{H}=\overrightarrow{0}$ under the superconducting surface it can be written that:

$$
\int_{S} \vec{j}_{s} d S=\int_{S}\left(\vec{n}_{s} \times \vec{H}_{/ /}\right) d S
$$

where $\vec{H}_{/ /}$is evaluated at $\mathrm{z}=0^{+}$(limit above the superconductor surface).

As this result is independent of the small parallelepiped previously chosen, the integrands must equal:

$$
\vec{j}_{s}=\vec{n}_{s} \times \vec{H}_{/ /}\left(z \rightarrow 0^{+}\right)
$$

Furthermore, $\vec{H}_{/ /}\left(z \rightarrow 0^{-}\right)=0$ and $\vec{H}_{/ /}^{s c}\left(z \rightarrow 0^{+}\right)=-\vec{H}_{/ /}^{s c}\left(z \rightarrow 0^{-}\right)$implies:

$$
\vec{H}_{/ /}^{s c}\left(z \rightarrow 0^{+}\right)=\vec{H}_{/ /}^{a p}\left(z \rightarrow 0^{+}\right)
$$

Therefore, an expression for the superconducting current as a function of the applied magnetic field may be written:

$$
\vec{j}_{s}=2 \vec{n}_{s} \times \vec{H}_{/ /}^{a p}=2 \vec{n}_{s} \times \vec{H}^{a p}
$$

All expressions shown use the MKS unit system.

Applying the divergence theorem clearly shows that the total charge is always conserved, for whichever surface shape the superconductor has, provided the source of the applied field is outside the superconductor:

$$
\int_{S} \vec{j}_{S} d S=\int_{S} 2 \vec{n}_{s} \times \vec{H}^{a p} d S=\int_{V} \vec{\nabla} \times \vec{H}^{a p} d^{3} x=\overrightarrow{0}
$$

Furthermore,

$$
\vec{H}_{\perp}=\vec{H}_{\perp}^{a p}+\vec{H}_{\perp}^{s c}=\overrightarrow{0}
$$


which is consistent with the previous expression for the superconducting current.

The total field thus generated for a semi-infinite plane is equivalent to that generated by the method of images (Cansiz \& Hull, 2005; Hellman et al. 1988; Hull \& Cansiz, 1999; Moon, 1994). As the expression (11) has been derived using only local arguments, it may be used for any shape of superconductor. It does not depend on the curvature of the surface.

\subsection{Force calculation}

The external force (by unit surface) experienced by the superconductor can be calculated by using Lorentz force.

$$
\frac{d \vec{F}}{d S}=\vec{j}_{s} \times \vec{B}^{a p}
$$

Using the previous expression for the superconducting current (1) and the constituent equation of air (15) (the medium in which the field is generated)

$$
\vec{B}^{a p}=\mu_{0} \vec{H}^{a p}
$$

it can be written that:

$$
\frac{d \vec{F}}{d S}=2 \mu_{0}\left(\vec{n}_{s} \times \vec{H}^{a p}\right) \times \vec{H}^{a p}
$$

This is a local and exact expression for the "pressure" or more precisely "stress" or force per unit surface on the superconductor, which depends only on the applied magnetic field. It is useful for any shape of superconductor. This differs fundamentally from the general expression used to calculate the magnetic stress between magnetic materials as given by Moon.

According to Newton's law, the force exerted by the superconductor on the magnet is simply the opposite one. Therefore, for any shape of superconductor, the force exerted by the superconductor on the magnet can be written as:

$$
\vec{F}=-\iint_{S c} 2 \mu_{0}\left(\vec{n}_{s} \times \vec{H}^{a p}\right) \times \vec{H}^{a p} d S
$$

where the integration extends over the whole surface of the superconductor.

\subsection{Torque calculation}

The torque suffered by the superconductor can easily be deduced as :

$$
\vec{M}_{S c}=\iint_{S c} \vec{r} \times\left(2 \mu_{0}\left(\vec{n}_{s} \times \vec{H}^{a p}\right) \times \vec{H}^{a p}\right) d S
$$

where $\vec{r}$ is the position vector between the differential surface element and the center of mass of the superconductor bulk. Again, the integration extends over the whole surface of the superconductor.

In order to calculate the moment applied over the magnet, the $\vec{r}_{P M}$ must be the position vector between the differential surface element and the center of mass of the permanent magnet. As noted previously, the force exerted by the superconductor on the external 
magnetic field (in this case a single permanent magnet) is simply the opposite one, and the same applies for the torque.

$$
\vec{M}_{P M}=-\iint_{S c} \vec{r}_{P M} \times\left(2 \mu_{0}\left(\vec{n}_{s} \times \vec{H}^{a p}\right) \times \vec{H}^{a p}\right) d S
$$

\section{Finite elements implementation}

Due to this differential form equation (16) can be easily implemented in a finite element program. A FEM algorithm has been adapted for the commercial software ANSYS. The SURF154 element of ANSYS was used insofar as it has defined a set of useful attributes e.g. the surface normal direction. The algorithm is valid in the context of a common electromechanical simulation. The steps for the simulation were:

- $\quad$ Select Element Type: SOLID98 (with a maximum of one degree of freedom MAG) and SURF154.

- Create the different materials to be used. For the superconductor bulk, air properties were used.

- Generate the geometries of the volumes for the electromagnetic system.

- Assign materials' properties to each volume, selecting air for the superconductor.

- Mesh the whole system with the SOLID98 element (as fine as is considered adequate discussed further below).

- $\quad$ Mesh the superconductor surface with the SURF154 element.

- Apply the electromechanical loads to the system.

- Solve the electromagnetic equation system.

Once the system has been solved, the algorithm can be applied using a Command List. Fig. 1 shows a flow-diagram of the procedure.

This procedure has to be performed for each piece of superconductor in the system. Should there be more than one piece, a different internal SURF154 element must be created and accordingly, the number of SURF154 elements in the first step (ESEL) must be changed.

These steps provide the three components of the force vector. The torque applied on the superconductor can also be calculated, from which the torques values can then be derived.

\subsection{Results provide by the post-processing}

The algorithm has been tested using one of the most common experiments found in relevant literature: a permanent magnet oriented vertically over a superconductor pile in any arbitrary position.

Firstly, an electromagnetic system composed of a small magnet suspended over a superconducting cylinder was designed, as shown in Fig. 2.

The dimensions of the superconductor were: $20 \mathrm{~mm}$ diameter and $7.5 \mathrm{~mm}$ height, and the small magnet was characterized by a coercivity of $875 \mathrm{kA} / \mathrm{m}$ and a remanence of $1.18 \mathrm{~T}$, with a $3.5 \mathrm{~mm}$ diameter and a $2 \mathrm{~mm}$ height. The magnet's centroid was placed $10 \mathrm{~mm}$ over the surface of the superconductor. The entire system was placed in surroundings measuring 100x100x100 mm.

The results that can be obtained are the distribution of forces, torque and current densities per surface element. In fig 3, these distributions for an arbitrary position of the magnet over the superconductor are shown. 


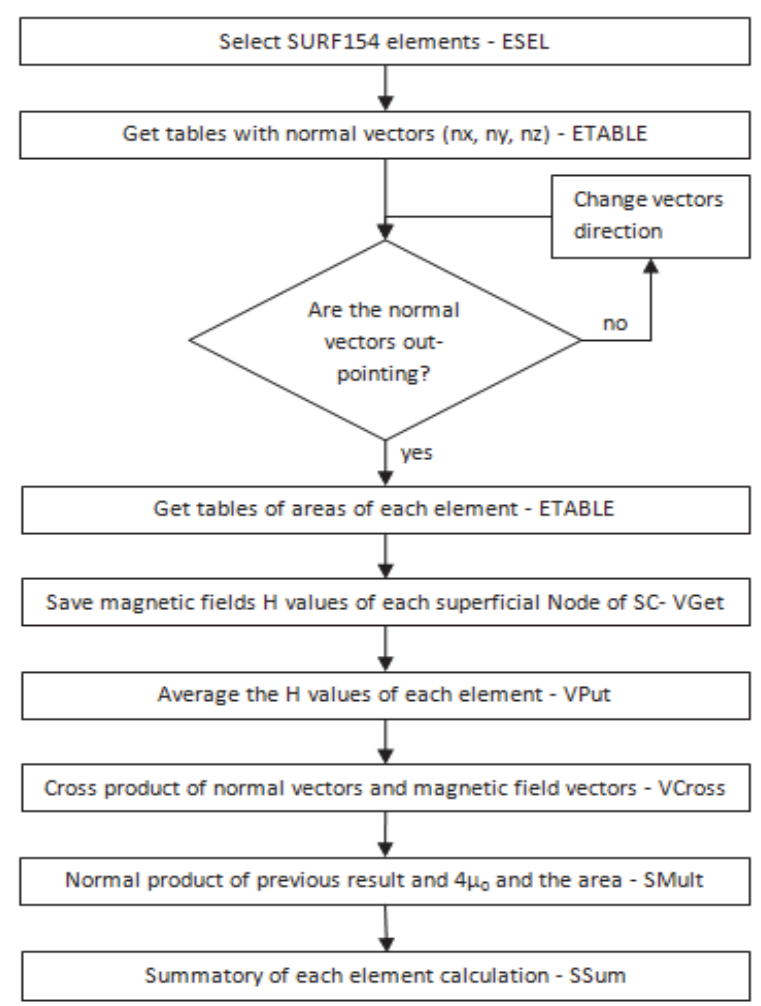

Fig. 1. Flow-diagram of the algorithm.

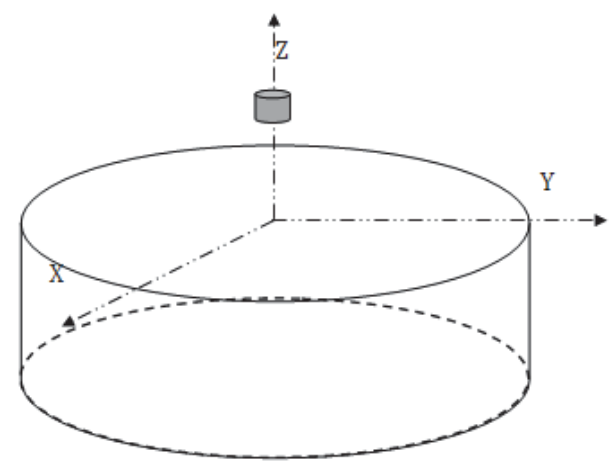

Fig. 2. Small permanent magnet $(\mathrm{m}=0.016 \mathrm{Am} 2)$ over superconductor. 


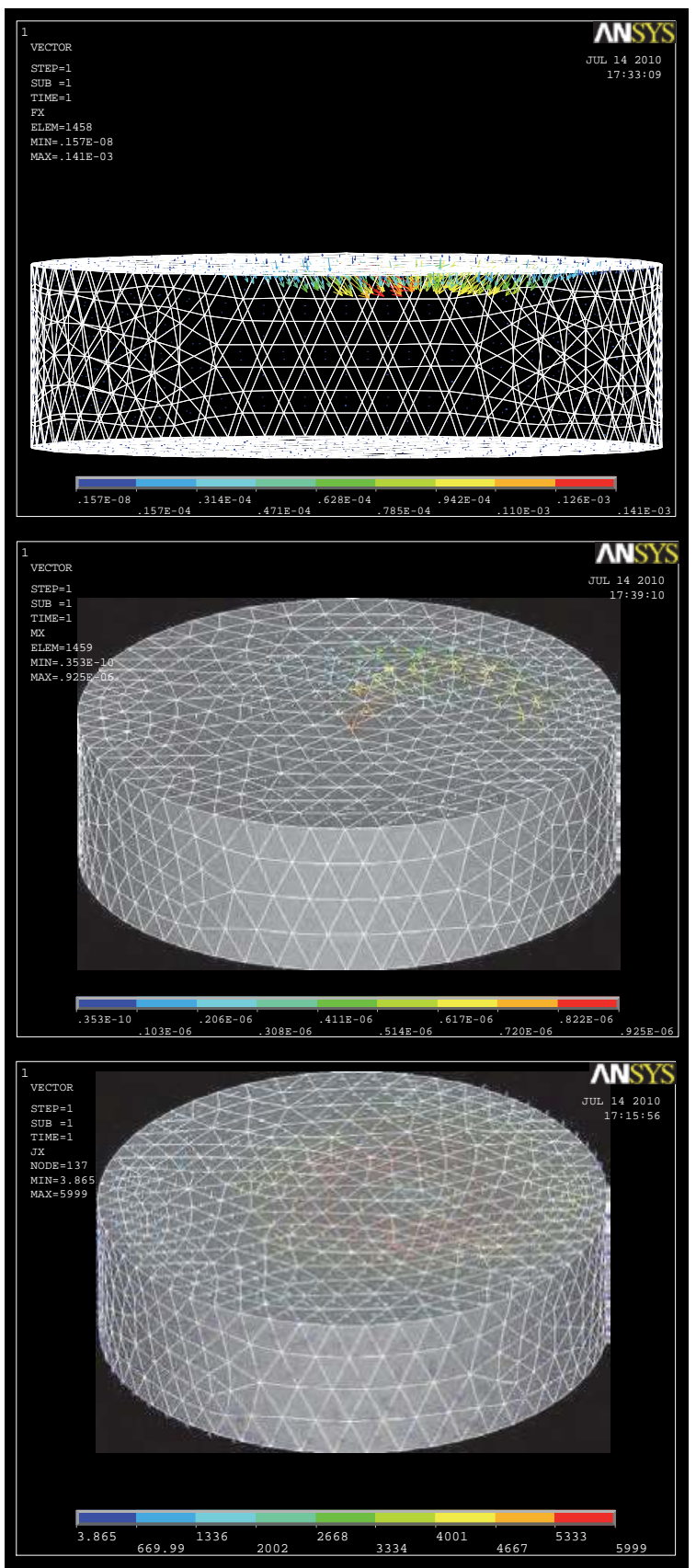

Fig. 3. Force, torque and current density distributions per surface element. 
The same simulation was repeated several times with different meshes, increasing the number of elements for the whole simulation. Using the parameter $\alpha$, the fineness of the mesh can be defined as the ratio between the maximum of the area of the elements and the total area of the superconductor multiplied by 100 .

$$
\alpha=\frac{\max (\text { elements areas) }}{\text { total SC area }} \times 100
$$

Different meshes along with their respective a parameters are shown in fig. 4 . The number of SURF154 elements and the values of the solution are also displayed.

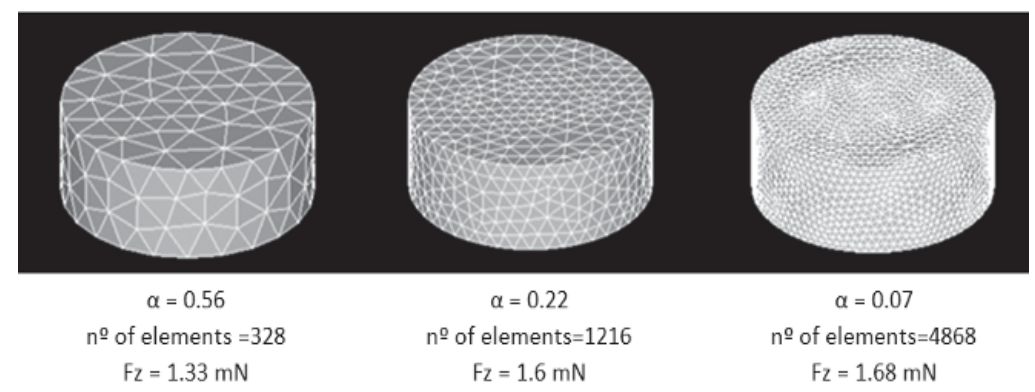

Fig. 4. Different meshes of the superconductor pile.

Fig. 5 shows the relative error of the calculations in relation to the analytical solution (with a magnetic moment of $0.016 \mathrm{~A} \mathrm{~m}^{2}$ ). The higher the number of surface elements, the smaller the relative error of the result. For example, where a is smaller than $0.1 \%$, the resulting relative error is less than $3 \%$.

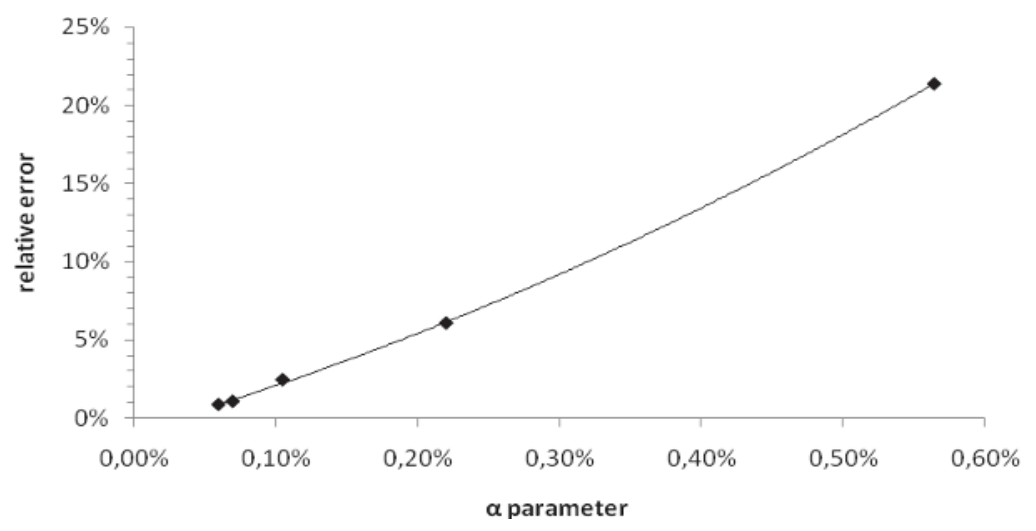

Fig. 5. Relative errors of the results vs. alpha for $\mathrm{z}=10 \mathrm{~mm}$.

In Fig. force versus $z$ are shown for different values of $a$. The FEM results tend towards the analytical values as a decreases. It is noted that the magnetic dipole approximation made for the analytical calculation only remains valid where there is a large distance between the permanent magnet and the superconductor. 


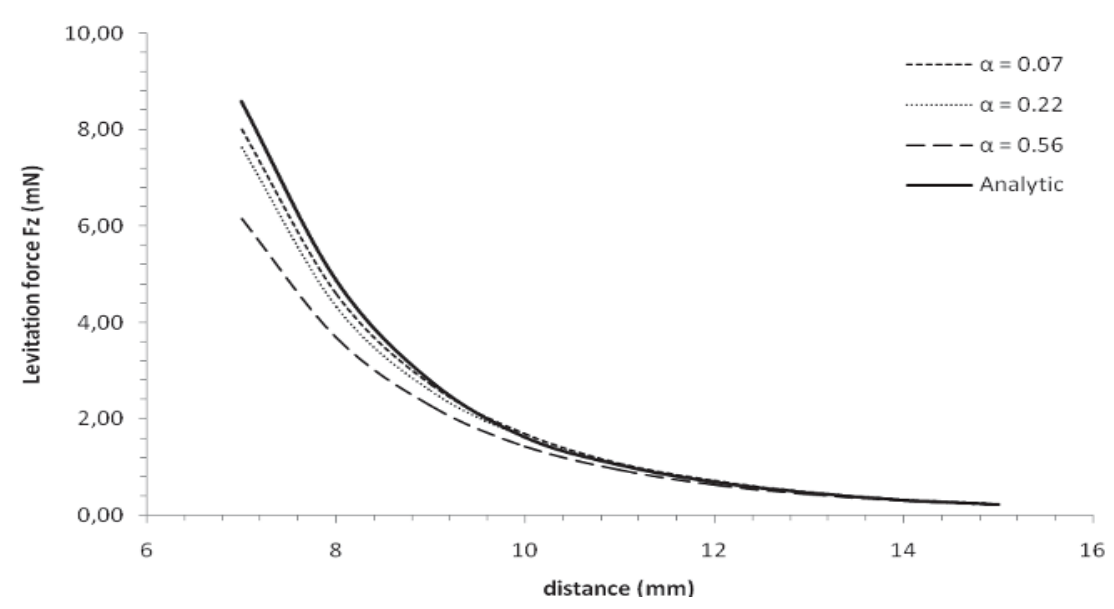

Fig. 6. Levitation force computed by the analytical expression with a point magnetic dipole and by FEM with different $a$.

The convergence of the algorithm has been checked in relation to the finite elements' size, and compared to analytical solutions for simple geometries. An a parameter has been proposed to assess the relative error in the results. The results showed good accuracy, whilst not requiring high specification computing technology.

\section{Experimental verification}

Different experiments were carried out in order to check the validity of the model. Some of them will be summarized in the following.

\subsection{Force measurement}

The following methodology was used to measure the forces: a cylindrical superconductor made of polycrystalline $\mathrm{YBa} 2 \mathrm{Cu} 3 \mathrm{O} 7-\mathrm{x}$, manufactured by CAN superconductors (Kamenice 25168, Czech Republic) was immersed in a bath of liquid nitrogen $\mathrm{N}_{2}(77 \mathrm{~K})$ at ambient pressure. The cylinder had a diameter of $45 \mathrm{~mm}$ and a height of $13 \mathrm{~mm}$. It was fixed to a nitrogen vessel. The vessel, containing the superconductor, was placed on a lab jack stand to adjust the height. A small cylindrical permanent magnet was used, which had a coercivity of $875 \mathrm{kA} / \mathrm{m}$, a remanence of $1.18 \mathrm{~T}$, and had a diameter of $5 \mathrm{~mm}$ and a height of $5 \mathrm{~mm}$. All experimental measurements followed the same coordinate system shown in Fig. 7. The origin of the coordinates was set at the center of the upper surface of the superconductor.

The permanent magnet was placed over the superconductor ( $Z$ coordinate), and fixed vertically to a PVC cantilever according to its magnetization direction $\left(\theta=90^{\circ}\right)$. The cantilever had 2 pairs of strain gauges to measure vertical forces at its extremes. This strain gauge configuration is not sensitive to the lateral and axial forces. The torques were neglected due to the size of the magnet. The PVC cantilever was joined to a 3D positioning table. The position of the magnet was then fixed in relation to the superconductor surface with a precision of $0.1 \mathrm{~mm}$. The strain gauges were calibrated using a dynamometer and a set of 12 references forces. The calibration constant was established by least squares fitting in $\mathrm{K}=(3.87 \pm 0.14) \times 10^{-4} \mathrm{~N} / \mu \varepsilon$, with a correlation coefficient of $\mathrm{R}^{2}=0.997$. 


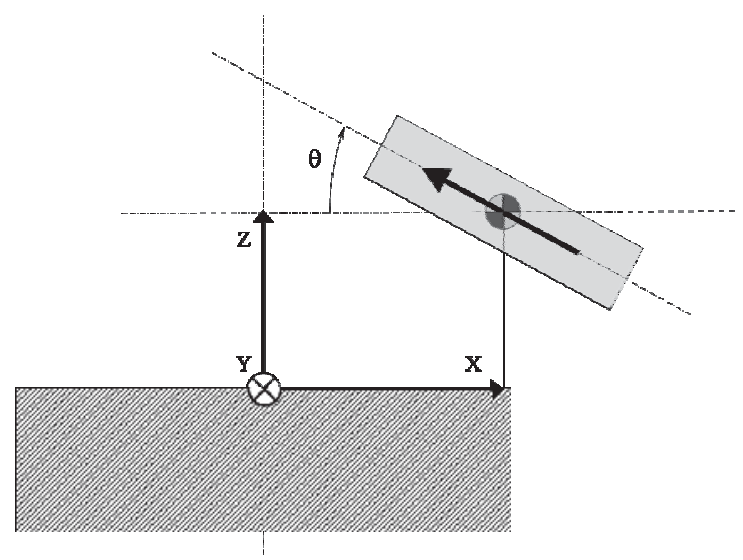

Fig. 7. Coordinate system of the PM-SC configuration. The superconductor is down and the permanent magnet is over it. Figure is not scaled to real sizes.

The measurement for every position was made in zero field cooling conditions (ZFC). The vertical forces were recorded where $X=0.0,5.0,10.0,15.0,17.5,20.0,22.5$ and $25.0 \pm 0.1 \mathrm{~mm}$; at 3 different heights from the surface of the superconductor: 12.0, 10.0, and $8.0 \pm 0.1 \mathrm{~mm}$. Furthermore, measurements were taken in the center of the upper superconductor face, $X=$ $0 \mathrm{~mm}$ from $\mathrm{Z}=7.0$ to $14.0 \pm 0.1 \mathrm{~mm}$. The $Y$ position was always fixed at $0 \mathrm{~mm}$.

These positions were chosen in order to avoid exceeding a limit of $3.5 \mathrm{mT}$ of magnetic flux density at any point of the superconductor surface. Using this limit ensures the Meissner state is retained. Regardless of this, after every measurement the remanent magnetization of the superconductor bulk was checked and in most cases no measurable magnetization was found. In order to compare the experimental and theoretical values, expression (16) was implemented in a finite element analysis program. The following figures (Fig 8-11) show the results.

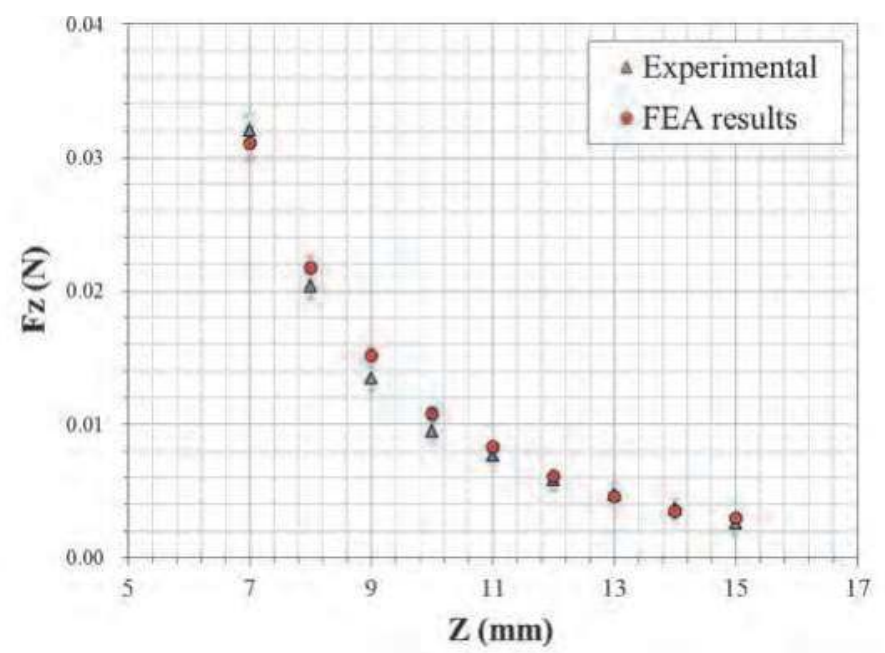

Fig. 8. $\mathrm{Z}$ dependence of vertical force for $\mathrm{X}=0 \mathrm{~mm}$. 
The FEA errors are estimated to be less than $3 \%$.

The figures show positive agreement between experimental and theoretical values. Only Fig. 9 shows an appreciable difference for values approaching $X=20 \mathrm{~mm}$. However, it must be pointed that the radius of the superconductor is $22.5 \mathrm{~mm}$. It is only in these surroundings that a very low remanent magnetization was recorded, which indicates a non complete Meissner state. This explains why some experimental values were lower than those of a complete Meissner state.

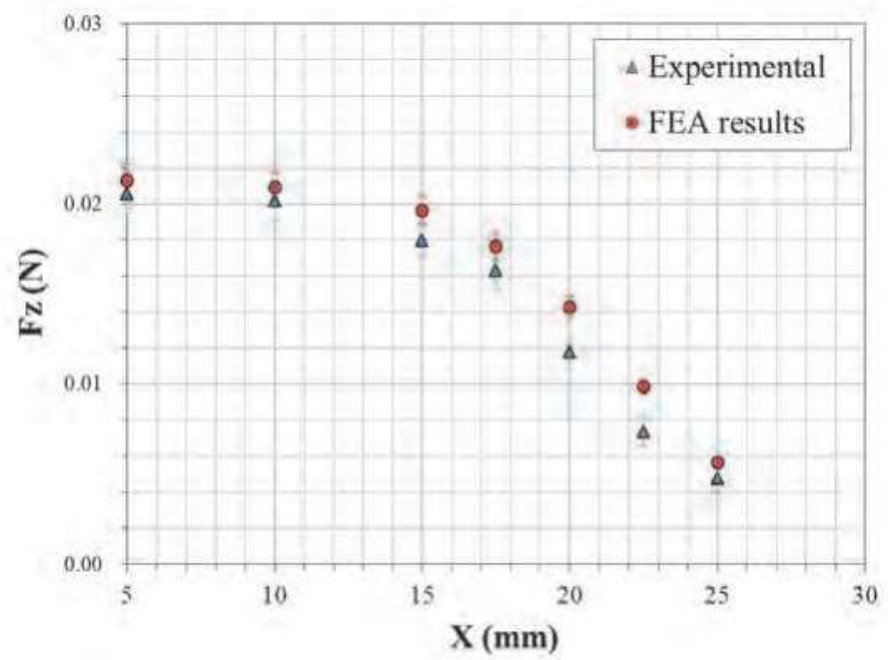

Fig. 9. $X$ dependence of vertical force for $Z=8 \mathrm{~mm}$.

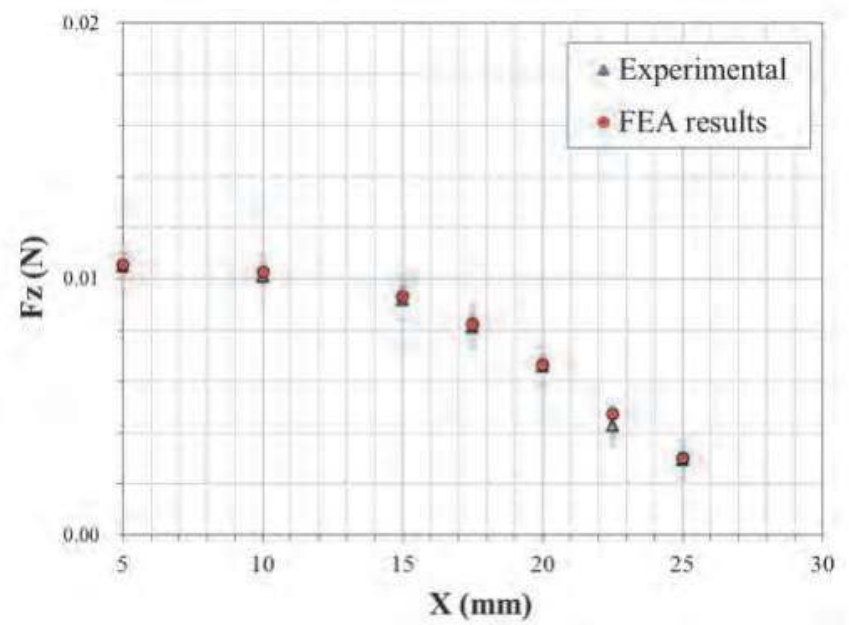

Fig. 10. $X$ dependence of vertical force for $Z=10 \mathrm{~mm}$. 


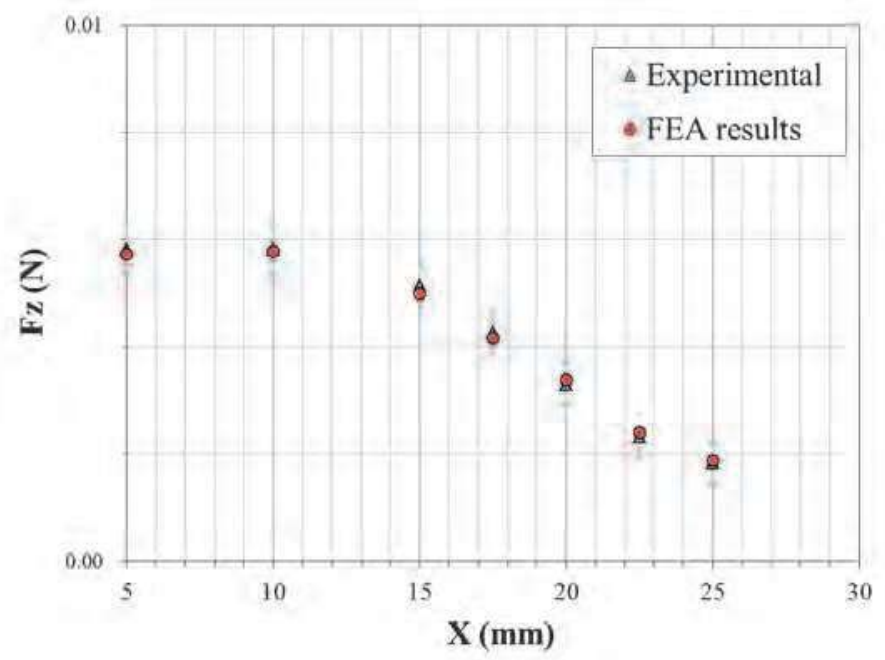

Fig. 11. $X$ dependence of vertical force for $Z=12 \mathrm{~mm}$.

\subsection{Equilibrium angle measurement}

In addition to previous experiments, the mechanical behavior of a magnet which has the ability to tilt over the superconductor in the Meissner state was also studied in this paper. In the present experiment only one degree of freedom was permitted in the tilt angle of the magnet ( $\theta$ coordinate). The equilibrium angle of the permanent magnet over the cylindrical superconductor was measured for different relative positions. The results can be used to understand not only how the permanent magnet is repelled, but also how it turns when it is released over a superconductor.

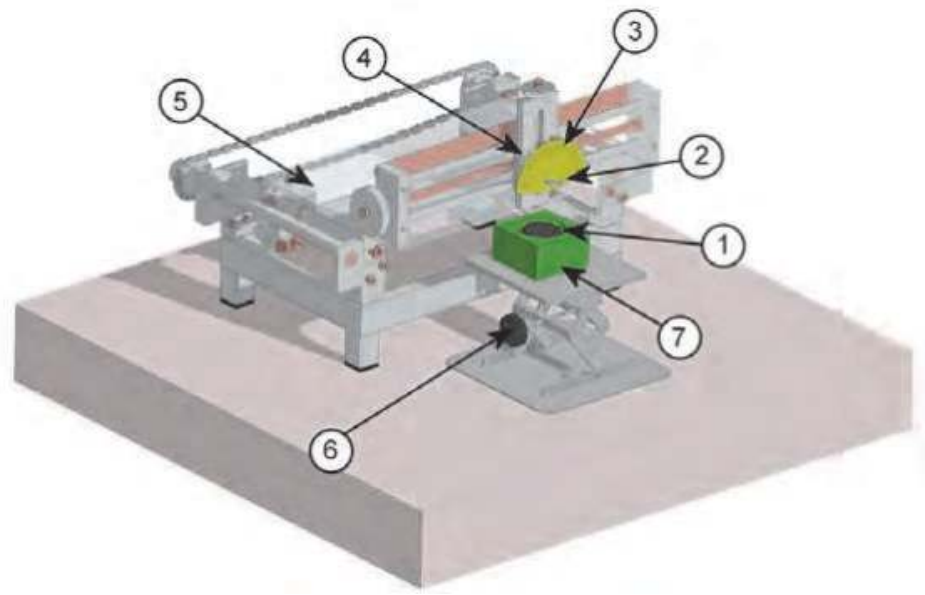

Fig. 12. Measurement system: 1 - Superconductor bulk, 2 - Permanent magnet, 3 Goniometer, 4 - Bearing (hidden), 5 - 3D table, 6 - Lab jack stand, 7 - Nitrogen vessel. 
A cylindrical permanent magnet (made of $\mathrm{NdFeB}$ with a coercivity of $875 \mathrm{kA} / \mathrm{m}$ and a remanence of $1.29 \mathrm{~T}$ ) was placed over the superconductor. Their dimensions were $6.3 \mathrm{~mm}$ in diameter and $25.4 \mathrm{~mm}$ in length and it had a magnetization direction parallel to its axis of revolution. A rigid plastic circular rod was fixed in the center of mass, perpendicular to the axis of revolution. This rod was used as the shaft in a plastic bearing, which was lubricated with oil. The whole bearing system was joined to a 3D displacement table. This arrangement ensured it was possible to control the position of the permanent magnet with an accuracy of $0.1 \mathrm{~mm}$, and the only permitted degree of freedom was the rotation around the $\mathrm{Y}$ axis. Concentric to the bearing, a graduate goniometer measured the angle of rotation of the magnet. The whole experiment design is shown in Fig 12.

Fig. 13 shows the comparison between the equilibrium angles measured and those calculated by expression (19).

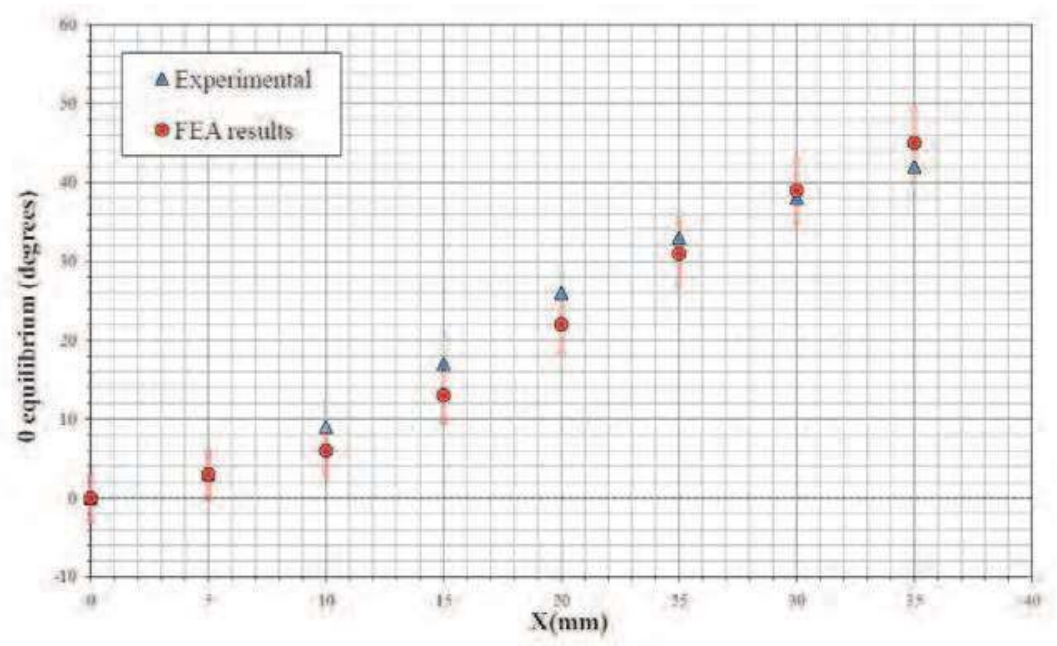

Fig. 13. Comparative graph between experimental and FEA calculus of the equilibrium angle versus $x$ position. Hight $z$ was fixed at $+15 \mathrm{~mm}$.

Again, there was a good agreement between the calculus made according to our model and the experiments. These experiments were carried out in Zero Field cooling condition (ZFC), and consequently there is no remanent magnetization.

\section{Limits of application}

The lower critical field, $H_{c 1}$, is one of the typical parameters of type II superconductors, which has been experimentally being assessed from the magnetization changes from the Meissner state slope to the reversible mixed-state behavior (Poole, 2007). $H_{c 1}$ is directly related to the free energy of a flux line and contains information on essential mixed-state parameters, such as the London penetration depth, $\lambda_{\mathrm{L}}$, and the Ginzburg-Landau parameter, к. Measurements of $H_{c 1}$ and, of course, of the upper critical field, $H_{c 2}$, therefore provide a complete characterization of the mixed-state parameters of the superconductor. 
Differences between the predicted Meissner forces and the experimentally measured ones indicate that a part of the sample is in the mixed-state. Establishing with precision the instant when the differences begin will permit us to determine the $H_{c 1}$ mechanically. Nevertheless, many other experimental techniques have been used to determine the state transition; most of them based on some kind of d.c. or a.c. magnetic measurement, but also on muon spin rotation ( $\mu S R)$ or magneto-optical techniques (Meilikhov \& Shapiro, 1992). The basic problem of magnetization measurements introduced by flux pinning lies in the fact that the change of slope at the lower critical field is extremely small, since the first penetrating flux lines are immediately pinned and change the overall magnetization $(M=m / V)$ only marginally. Elaborate schemes of subtracting the measured moments from an initial Meissner slope (Vandervoort et al., 1991; Webber et al., 1983) or experiments providing us directly the derivative of magnetization (Hahn \& Weber, 1983; Wacenoysky et al., 1989; Weber et al., 1989) have been employed, SQUIDS have also been used to improve the precision of these kind of means (Böhmer et al., 2007).

The method also determines the zone at the sample where transition from Meissner to mixed state occurs.

For a position of the magnet with respect to the superconductor we define the Meissner Efficacy as

$$
\eta=\frac{F_{e x}}{F_{M}}
$$

where $F_{e x}$ is the experimentally measured force and $F_{M}$ is the calculated force according with the Meissner model cited above. For a certain position of the magnet a Meissner Efficacy equal to one $(\eta=1)$ proves that the superconductor is completely in the Meissner state and there is not any flux penetration. On the contrary, values lower than 1 indicate that a part of the superconductor has flux penetration and is in the mixed-state.

The measurement for every position was made in zero field cooling conditions (ZFC). The origin of coordinates was set at the center of the upper surface of the superconductor. The reference point of the magnet was placed in the center of the lower surface of the magnet. Therefore, the $\mathrm{Z}$ coordinate is the distance between the faces of the magnet and the superconductor. $X$ is the distance of the center of the magnet to the axis of the superconductor cylinder (radial position). We have recorded the vertical forces for $X=0.0$, 5.0, 10.0, 15.0, 17.5, 20.0, 22.5 and $25.0 \pm 0.1 \mathrm{~mm}$; at 4 different heights from the surface of the superconductor: $12.0,10.0,8.0$ and $6.0 \pm 0.1 \mathrm{~mm}$.

Fig. 14 shows the Meissner Efficacy versus the maximum of the surface current density distribution $J_{\text {surf }}$ for different positions.

We observe that for low values of the maximum surface current density, the Meissner Efficacy is just 1 .

From a certain value, the Meissner Efficacy decays linearly. From this data we can derive a weighted mean value of $J_{c 1 \text { surf }}=6452 \pm 353 \mathrm{~A} / \mathrm{m}$ for a polycrystalline $\mathrm{YBa}_{2} \mathrm{Cu}_{3} \mathrm{O}_{7-\mathrm{x}}$ sample at $77 \mathrm{~K}$.

In Table $1 H_{c 1}$ values from different authors are shown for comparison. The values are those obtained for the $H_{c 1}$ parallel to c-axis in monocrystalline samples. Our value for a polycrystalline sample is of the same order of magnitude than the lowest monocrystalline values. 


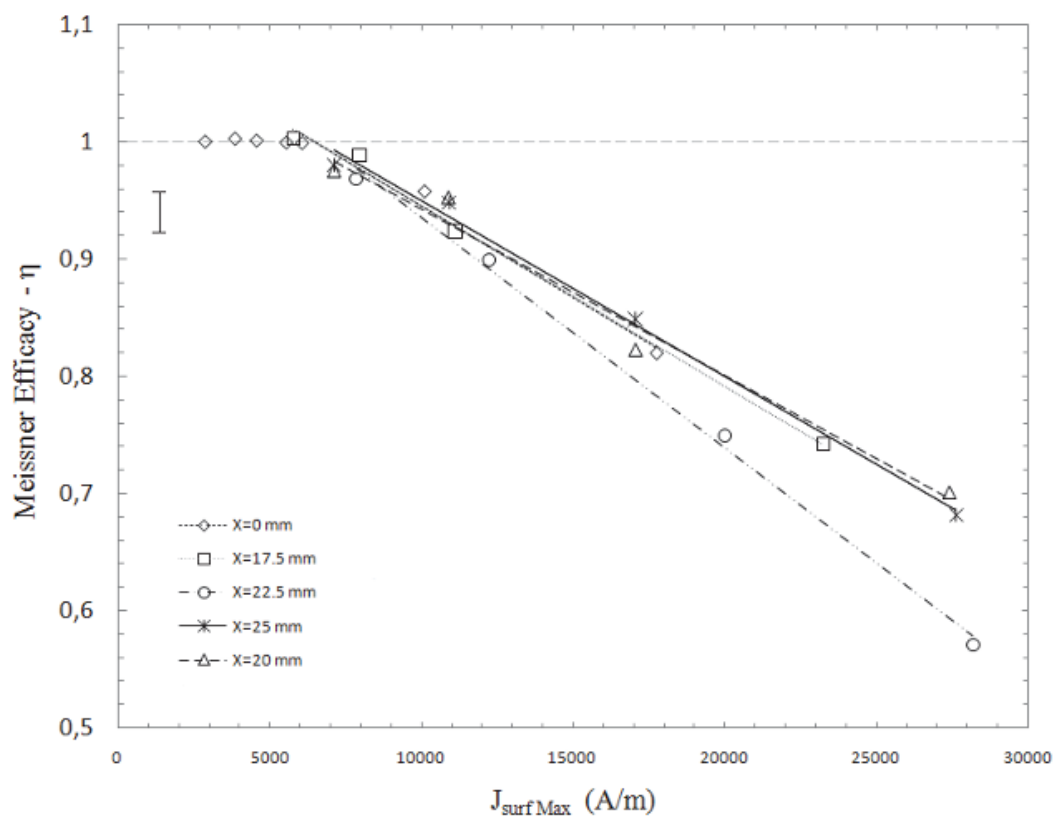

Fig. 14. Meissner Efficacy versus maximum Jsurf for different positions. The values obtained for $X=5.0,10.0,15.0 \mathrm{~mm}$ radial positions are similar to those obtained for the $X=0.0 \mathrm{~mm}$ values.

Now, if we use a value of $\lambda_{L}=4500 \AA$, carried out from the literature (Geflbaux \& Tazawa, 1998; Mayer \& Schuster, 1993) we have a lower critical current density value of $J c_{1}=(1.43 \pm$ $0.08) \times 10^{7} \mathrm{~A} / \mathrm{cm}^{2}$. By using Eq. 2 we calculate $H c_{1}=3226 \pm 176 \mathrm{~A} / \mathrm{m}$.

\begin{tabular}{c|c|c|c|c|c}
\hline \hline & $\begin{array}{c}\text { C. Bömer et al } \\
(2007) \\
\text { (monocr.) }\end{array}$ & $\begin{array}{c}\text { Umewaza et } \\
\text { al (2007) } \\
\text { (monocr.) }\end{array}$ & $\begin{array}{c}\text { Kaiser et al } \\
(1991) \\
\text { (monocr.) }\end{array}$ & $\begin{array}{c}\text { Wu et al } \\
(1990) \\
\text { (monocr.) }\end{array}$ & $\begin{array}{c}\text { Mechanical } \\
\text { method } \\
\text { (polycr.) }\end{array}$ \\
\hline $\begin{array}{c}\text { Results for } H_{c 1} \\
(\mathrm{~A} / \mathrm{m})\end{array}$ & $\begin{array}{c}2900 \pm 250 \\
6000 \pm 2300\end{array}$ & $4500 \pm 450$ & $\begin{array}{c}3580 \\
11000\end{array}$ & $\begin{array}{c}4950 \\
15518\end{array}$ & $3226 \pm 176$ \\
\hline
\end{tabular}

Table 1. Comparison of the values found in different articles with that measured in this paper. The values and relative errors have been obtained directly from graphs, at $77 \mathrm{~K}$. Available values for $\mathrm{H} \perp(\mathrm{a}, \mathrm{b})$ and $\mathrm{H} \|$ (c) in monocrystals are shown. $\mathrm{H} \|$ (c) is always greater than $\mathrm{H} \perp(\mathrm{a}, \mathrm{b})$

The uncertainty in the determination of $J_{c 1}$ surf may be reduced by increasing the number of series of measurements (or paths). Therefore, this is a method intrinsically more precise than other common methods.

In fact, the values far from the Meissner state contribute to improve the accuracy of the $J_{c 1}$ surf determination. The determination of the slopes of straight lines has a propagation of errors 
more convenient than that in the case of the measurement of a change in the slope of the tangents to a curve. Other methods, therefore, would require high precision measurements to obtain a reasonable error for Hc1.

This results are in according to the border and thickness effects and border magnetization that have been already described by other authors in an uniform magnetic field (Brandt, 2000; Morozov et al., 1996; Li et al., 2004; Schmidt et al., 1997):

\section{Example of application - permanent magnet over a superconducting torus}

We calculate the torque exerted between a superconducting torus and a permanent magnet by using this model. We find that there is a flip effect on the stablest direction of the magnet depending on its position. This could be easily used as a digital detector for proximity.

We consider a full superconducting torus and a cylindrical permanent $\mathrm{NdFeB}$ magnet over the superconductor axis ( $\mathrm{Z}$ axis). In figure 15 we can observe the geometrical configuration of both components. Every calculation is referenced with respect of a Cartesian coordinate system placed in the center of mass of the torus which $\mathrm{Z}$ axis is coincident with the axis of the torus.

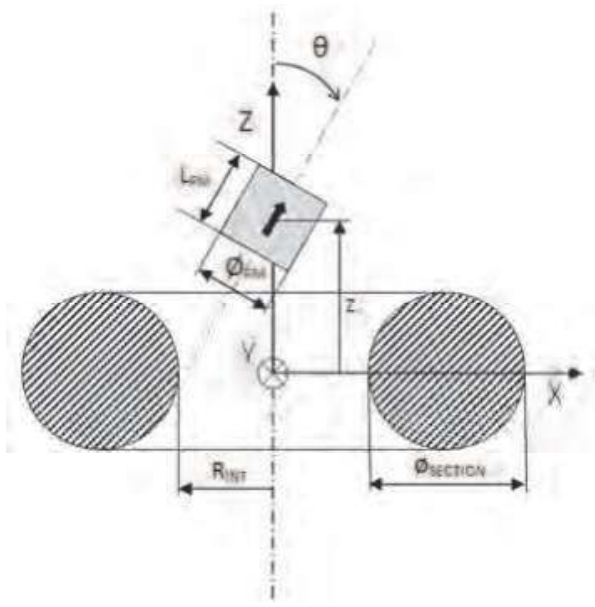

Fig. 15. Permanent magnet over a toroidal superconductor set-up. The dimensions are: $\mathrm{L}_{\mathrm{PM}}$ length of the cylindrical permanent magnet, $\varnothing_{\mathrm{PM}}$ - diameter of the cylindrical permanent magnet, $R_{I N T}$ - Inner radius of the torus, $\varnothing_{\mathrm{SECTION}}$ - Diameter of the circular section of the torus. $\mathrm{z}$ is the vertical coordinate of the center of the magnet and $\theta$ is the angle between the axis of the magnet and the vertical $\mathrm{Z}$ axis.

The superconducting torus has an internal radius $\mathrm{R}_{\mathrm{INT}}=6 \mathrm{~mm}$ and a diameter of the section $\varnothing_{\text {SECTION }}=10 \mathrm{~mm}$. The cylindrical permanent magnet has a length $\mathrm{L}_{\mathrm{PM}}=5 \mathrm{~mm}$ and a diameter $\varnothing_{\mathrm{PM}}=5 \mathrm{~mm}$. When calculating the magnetic field generated by the magnet we define its magnetic properties as: Coercive magnetic field $\mathrm{H}_{\text {COERCIVITY }}=875 \mathrm{kA} / \mathrm{m}$ and remanent magnetic flux density $B_{\text {REMANENT }}=1.18 \mathrm{~T}$. We assume that the direction of magnetization of the permanent magnet coincides with its axis of revolution.

The variables $\theta$ and $z$ are the coordinates we modify in order to analyze the mechanical behavior of the magnet over the superconductor. $\mathrm{z}$ is the distance along the $\mathrm{Z}$ axis between 
the center of mass of the torus and the one of the cylindrical permanent magnet. $\theta$ is the angle between the axis of the magnet and the vertical $\mathrm{Z}$ axis.

The equilibrium angle $\left(\theta_{\text {eq }}\right)$ as a function of $\mathrm{z}$ can be determined as follows. For a certain $\mathrm{z}$ we calculate the $\mathrm{Y}$ component of the torque $\left(\mathrm{M}_{\mathrm{y}}\right)$ exerted on the magnet by the superconductor as a function of $\theta$ and we find the equilibrium angle as the value for which $\mathrm{M}_{\mathrm{y}}\left(\theta_{\mathrm{eq}}\right)=0$. The sign of the slope $\mathrm{dM}_{\mathrm{y}} / \mathrm{d} \theta$ at that point determines the stability or instability of the equilibrium point.

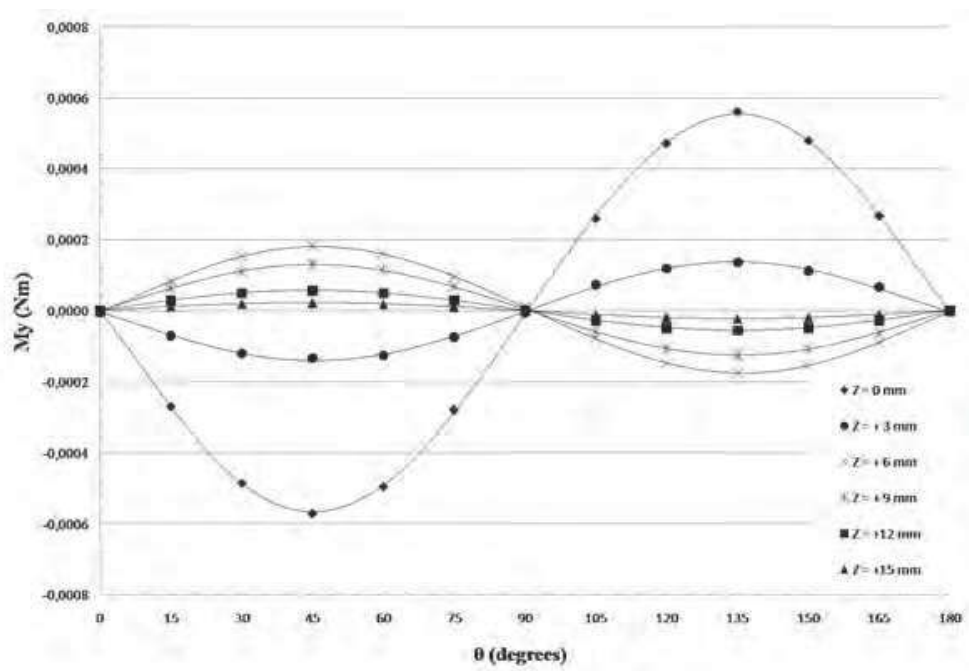

Fig. 16. $\mathrm{M}_{\mathrm{y}}$ applied to the permanent magnet by the superconductor as a function of $\theta$ for $\mathrm{z}=$ $0,3,6,9,12$ and $15 \mathrm{~mm}$.

In figure 16 the torque $\left(\mathrm{M}_{\mathrm{y}}\right)$ exerted on the magnet by the superconductor as a function of $\theta$ is shown for $\mathrm{z}=0,3,6,9,12$ and $15 \mathrm{~mm}$. The maximum values for the torque exerted to the permanent magnet appear at $\theta=45^{\circ}$ and $\theta=135^{\circ}$ for every $z$. The remarkable fact is that the sign suddenly changes when moving from $\mathrm{z}=3 \mathrm{~mm}$ to $\mathrm{z}=6 \mathrm{~mm}$. The equilibrium points are always at $\theta=0^{\circ}$ and $\theta=90^{\circ}$, but $\theta=0^{\circ}$ is a stable equilibrium point for $\mathrm{z}=0 \mathrm{~mm}$ and $\mathrm{z}=$ $3 \mathrm{~mm}$, while it is unstable for the rest of the positions. On the other hand $\theta=90^{\circ}$ is unstable for $\mathrm{z}=0 \mathrm{~mm}$ and $\mathrm{z}=3 \mathrm{~mm}$, but it is stable for the rest of the positions. That means that if you approach a magnet along the $\mathrm{Z}$ axis and it is able to rotate, it will be perpendicular to the $\mathrm{Z}$ axis while it is at $\mathrm{z} \geq 6 \mathrm{~mm}$, but it will suddenly rotate to be parallel to the $\mathrm{Z}$ axis when you pass from $\mathrm{z}=6$ to $\mathrm{z} \leq 3$.

In figure 17 the variation of the torque at $\theta=45^{\circ}$ as a function of $z$. The torque changes its sign between $\mathrm{z}=3 \mathrm{~mm}$ and $\mathrm{z}=4 \mathrm{~mm}$.

Finally, figure 18 shows the stable equilibrium angle as a function of $\mathrm{z}$. It is evident that, at a certain position between $\mathrm{z}=+3$ and $\mathrm{z}=+4 \mathrm{~mm}$ we found that the stable equilibrium angle switches from a vertical orientation of the magnet to an horizontal one describing the flip effect claimed in this work.

Therefore, it can be concluded that if you approach a magnet along the $\mathrm{Z}$ axis and it is able to rotate, it will be perpendicular to the $Z$ axis while it is at a certain distance $(z \geq 4 \mathrm{~mm}$ in 
our example) and it will change to be parallel to the $Z$ axis for closer positions ( $z \leq 3 \mathrm{~mm}$ in our example). As the equilibrium angle does not depend on the magnetic moment, the magnet can be much smaller. As a flip in the orientation of a permanent magnet can be easily instrumented, this effect can be easily used as a binary detector for proximity.

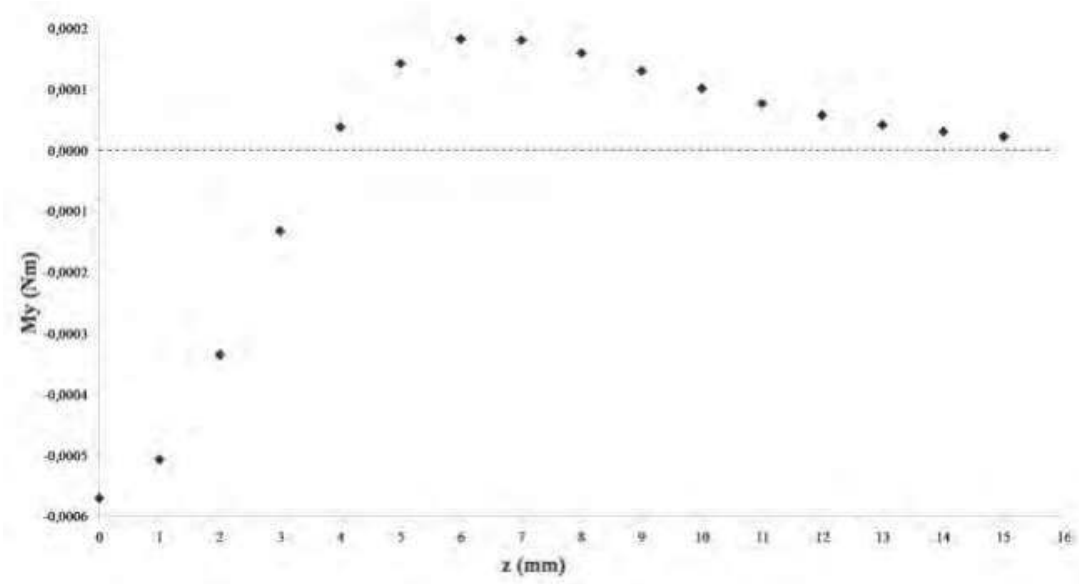

Fig. 17. Torque $M_{y}$ exerted on the magnet for $\theta=45^{\circ}$ as a function of $z$.

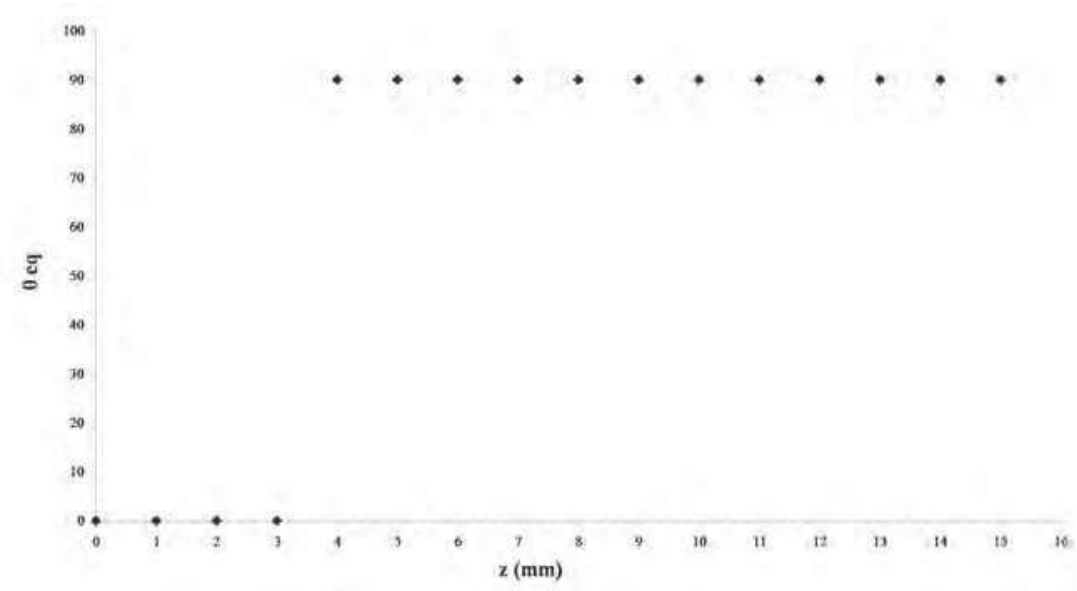

Fig. 18. Stable equilibrium angle $\left(\theta_{\mathrm{eq}}\right)$ as a function of $\mathrm{z}$.

\section{Conclusion}

Magnet-superconductor forces both in Meissner and mixed states can be calculated with the accuracy required to engineer useful levitating devices.

The implementation of a local differential expression in a finite elements program opens new perspectives to the use of magnet-superconductor devices for engineering. This can be 
used to calculate forces whatever the size, shape and geometry of the system, for both permanent magnets and electromagnets.

Accuracy and convergence, in addition to the experimental verification for different cases have been tested. There is a good agreement between experimental results and calculation, even with very low-cost computing resources involved.

Moreover, the expression can be used to determine the point when the mixed state arises in a superconductor piece.

\section{References}

Alario, M.A. \& Vicent, J.L. (1991). Superconductividad, 1st ed. (EUDEMA, Madrid, 1991).

Arkadiev, V. (1947). A floating magnet. Nature 160, 330 (1947).

Bednorz, J.G. \& Müller, K.A. (1986). Possible highT c superconductivity in the Ba- La- Cu- O system. Zeitschrift Für Physik B Condensed Matte.r 64, 189-193 (1986).

Böhmer, C.; Brandstatter, G. \& Weber, H. (2007). The lower critical field of high-temperature superconductors. Supercond. Sci. Techn., 10 (2007) A1-A10

Brandt, E.H. (2000). Superconductors in realistic geometries: geometric edge barrier versus pinning. Physica C, 332 (2000) 99-107

Buchhold, T.A. (1960). Applications of superconductivity. Scientific American 3, 74 (1960).

Cansiz, A.; Hull, J.R. \& Gundogdu O. (2005). Translational and rotational dynamic analysis of a superconducting levitation system. Supercond. Sci. Technol. 18, 990 (2005).

Diez-Jimenez E. et al. (2010). Finite element algorithm for solving supercondcuting Meissner repulsion forces. Icec 23 - Icmc 2010 (Wroclaw (Poland), 2010).

Early, E.A.; Seaman, C.L.; Yang, K.N. \& Maple, M.B. (1988) American Journal Of Physics 56, 617 (1988).

Geflbaux, X. \& Tazawa, M. (1998). Étude de la variation de la longueur de London entre 5 et $70 \mathrm{~K}$, dans un film très mince $\mathrm{d}$ ' $\mathrm{YBaCuOç6}$, par spectromérie dans I ' infrarouge lointain ; comparaison avec NbN. C.R. de l'Academie Sciences. Fasc. B, 324 (1998) 389397

Giaro, K.; Gorzkowski, W. \& Motylewski, T. (1990). A correct description of the interaction between a magnetic moment and its image. Physica C 168, 479-481 (1990).

Gijutsucho, K. (2001) Patent JP2001004652-A, (2001) Japan

Hahn, P. \& Weber, H.W. (1983). Automatic device for magnetization measurements on superconductors. Cryogenics, 23 (1983) 87-90

Hellman F. et al. (1988). Levitation of a magnet over a flat type II superconductor. Journal Of applied Physics 63, 447 (1988).

Hull, J.R. \& Cansiz, A. (1999). Vertical and lateral forces between a permanent magnet and a high-temperature superconductor. Journal Of Applied Physics 86, (1999).

Hull, J.R. (2000). Superconducting bearings. Supercond. Sci. Techn., 13 (2000) R1-R15

Jackson, J.D. (1975) Classical Electrodynamics (John Wiley \& Sons, New York, 1975).

Kaiser, D.L. \& Swartzendruber, L.J. (1991). Lower critical field measurements in YBACUO single cristals. Proc. Adv. in mat. sci. E appl. of HTSC (1991)

Li, Q.; Suenaga, M. \& Ye, Z. (2004). Crossover of thickness dependence of critical current density J(c)(T,H) in YBa2Cu3O7-delta thick films. App. Physics Lett. 84 (2004) 35283530

Lin, Q. (2006). Theoretical development of the image method for a general magnetic source 
in the presence of a superconducting sphere or a long superconducting cylinder. Physical Review B 74, 24510 (2006).

Mayer, B. \& Schuster, S. (1993). Magnetic-field dependence of the critical current in yba2cu3o7-delta bicrystal grain-boundary junctions. App. Physics Lett. 63 (1993) 783-785

Meilikhov, E. \& Shapiro, V.G. (1992). Critical fields of the htsc superconductors. Supercond. Sci. Techn., 5 (1992) S391-S394

Moon, F.C. (1994). Superconducting Levitation (John Wiley \& Sons, New York, 1994).

Morozov, N. \& Zeldov, E. (1996). Negative local permeability in Bi2Sr2CaCu2O8 crystals. Phys. Rev. Lett. 76 (1996) 138-141

Perez-Diaz, J.L. \& Garcia-Prada, J.C. (2007). Finite-size-induced stability of a permanent magnet levitating over a superconductor in the Meissner state. Applied Physics Letters 91, 142503 (2007).

Perez-Diaz, J.L. \& Garcia-Prada, J.C. (2007). Interpretation of the method of images in estimating superconducting levitation. Physica C 467, 141-144 (2007).

Perez-Diaz, J.L.; Garcia-Prada, J.C. \& Diaz-Garcia, J.A. (2008). Universal Model for Superconductor-Magnet Forces in the Static Limit. I.R.E.M.E. 2, (2008).

Poole, C.P. (2007) Superconductivity, 2nd ed.,AP, Elsevier, 2007

Schmidt, B.; Morozov, N. \& Zeldov, E. (1997). Angular dependence of the first-order vortexlattice phase transition in Bi2Sr2CaCu2O8. Phys. Rev. B, 55 (1997) R8705-R8708

Simon, I. (1953). Forces acting on superconductors in magnetic fields. Journal Of Applied Physics 24, 19 (1953).

Umezawa, A. \& Crabtree, A. (1998). Anisotropy of the lowr critical field, magnetic penetration depth, and equilibrium shielding current y single-crystal YBACUO. Physical Review B. 38, (1998).

Vandervoort, K.G.; Welp, U. \& Kessler, J.E. (1991). Magnetic measurements of the upper and lower critical fields of oxygen-deficient yba2cu3o7-delta single-crystals. Phys. Rev. B, 43 (1991) 13042-13048

Wacenovsky, M.; Weber, H.; Hyun, O.B. (1989). Magnetization of grain-aligned hoba2cu3o7delta. Physica C, 162 (1989) 1629-1630

Weber, H.W.; Seidl, E. \& Laa, C. (1991). Anisotropy effects in superconducting niobium. Phys. Rev. B, 44 (1991) 7585-7600

Wu, D.H. \& Sridhar, S. (1990). Pinning forces and lower critical fields in YBACUO crystals: temperature dependence and anisotropy. Phys. Rev. Lett. 65 (1990) 2074-2077

Yang, Y. \& Zheng, X. (2007). Method for solution of the interaction between superconductor and permanent magnet. Journal Of Applied Physics 101, 113922 (2007). 


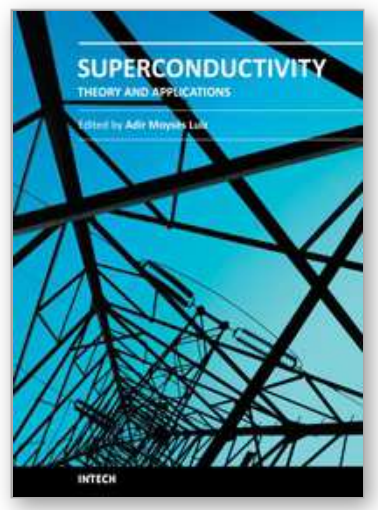

\section{Superconductivity - Theory and Applications}

Edited by Dr. Adir Luiz

ISBN 978-953-307-151-0

Hard cover, 346 pages

Publisher InTech

Published online 18, July, 2011

Published in print edition July, 2011

Superconductivity was discovered in 1911 by Kamerlingh Onnes. Since the discovery of an oxide superconductor with critical temperature (Tc) approximately equal to $35 \mathrm{~K}$ (by Bednorz and Müller 1986), there are a great number of laboratories all over the world involved in research of superconductors with high Tc values, the so-called â€œHigh-Tc superconductorsâ€. This book contains 15 chapters reporting about interesting research about theoretical and experimental aspects of superconductivity. You will find here a great number of works about theories and properties of High-Tc superconductors (materials with Tc $>30 \mathrm{~K}$ ). In a few chapters there are also discussions concerning low-Tc superconductors ( $\mathrm{Tc}<30 \mathrm{~K}$ ). This book will certainly encourage further experimental and theoretical research in new theories and new superconducting materials.

\section{How to reference}

In order to correctly reference this scholarly work, feel free to copy and paste the following:

Jose Luis Perez-Diaz and Efren Diez-Jimenez (2011). Foundations of Meissner superconductor magnet mechanismus engineering, Superconductivity - Theory and Applications, Dr. Adir Luiz (Ed.), ISBN: 978-953307-151-0, InTech, Available from: http://www.intechopen.com/books/superconductivity-theory-andapplications/foundations-of-meissner-superconductor-magnet-mechanismus-engineering

\section{INTECH}

open science | open minds

\author{
InTech Europe \\ University Campus STeP Ri \\ Slavka Krautzeka 83/A \\ 51000 Rijeka, Croatia \\ Phone: +385 (51) 770447 \\ Fax: +385 (51) 686166 \\ www.intechopen.com
}

\author{
InTech China \\ Unit 405, Office Block, Hotel Equatorial Shanghai \\ No.65, Yan An Road (West), Shanghai, 200040, China \\ 中国上海市延安西路65号上海国际贵都大饭店办公楼 405 单元 \\ Phone: +86-21-62489820 \\ Fax: +86-21-62489821
}


(C) 2011 The Author(s). Licensee IntechOpen. This chapter is distributed under the terms of the Creative Commons Attribution-NonCommercialShareAlike-3.0 License, which permits use, distribution and reproduction for non-commercial purposes, provided the original is properly cited and derivative works building on this content are distributed under the same license. 